ARTICLE

Received 28 Aug 2014 | Accepted 18 Feb 2015 | Published 25 Mar 2015

DOI: $10.1038 /$ ncomms 7677

OPEN

\title{
Ferromagnetism induced by entangled charge and orbital orderings in ferroelectric titanate perovskites
}

\author{
N.C. Bristowe ${ }^{1,2}$, J. Varignon ${ }^{1}$, D. Fontaine ${ }^{1}$, E. Bousquet ${ }^{1} \&$ Ph. Ghosez $^{1}$
}

In magnetic materials, the Pauli exclusion principle typically drives anti-alignment between electron spins on neighbouring species resulting in antiferromagnetic behaviour. Ferromagnetism exhibiting spontaneous spin alignment is a fairly rare behaviour, but once materialized is often associated with itinerant electrons in metals. Here we predict and rationalize robust ferromagnetism in an insulating oxide perovskite structure based on the popular titanate series. In half-doped layered titanates, the combination of Jahn-Teller and oxygen breathing motions opens a band gap and creates an unusual charge and orbital ordering of the $\mathrm{Ti} d$ electrons. It is argued that this intriguingly intricate electronic network favours the elusive inter-site ferromagnetic (FM) ordering, on the basis of intra-site Hund's rules. Finally, we find that the layered oxides are also ferroelectric with a spontaneous polarization approaching that of $\mathrm{BaTiO}_{3}$. The concepts are general and design principles of the technologically desirable FM ferroelectric multiferroics are presented.

\footnotetext{
${ }^{1}$ Theoretical Materials Physics, University of Liège, B-4000 Sart Tilman, Belgium. ${ }^{2}$ Department of Materials, Imperial College London, London SW7 2 AZ, UK. Correspondence and requests for materials should be addressed to N.C.B. (email: n.bristowe@imperial.ac.uk) or to Ph.G. (email: Philippe.ghosez@ulg.ac.be).
} 
$\mathrm{P}$ erovskite oxides exhibit a fascinating range of physical properties, including ferroelectricity, (anti)ferromagnetism (AFM), superconductivity and magnetoresistance. This diverse behaviour is appealing for both fundamental and applied investigations, and has resulted in an intense global research effort over the past few decades. Many of these functional properties manifest due to the complex and subtle interplay between spin, charge, orbital and lattice degrees of freedom in perovskites $^{1-4}$. Of the perovskites, the doped manganites have become a prototypical playground for the study of this interplay. Just considering the case of half-doping, that is, $\mathrm{A}_{0.5}^{2+} \mathrm{R}_{0.5}^{3+} \mathrm{MnO}_{3}$, where $\mathrm{A}^{2+}$ is a divalent alkaline earth metal ion and $\mathrm{R}^{3+}$ is a trivalent rare earth ion, manganites exhibit a rich variety of electronic phases. For example, half-doped manganites can display ferromagnetic (FM) or A-type AFM metallic behaviour ${ }^{5-7}$ or more commonly a CE-type AFM Mott insulating phase 8 ,9 associated with two different charge orderings (rocksalt ${ }^{10}$ and columnar ${ }^{11}$ ) and two different orbital orderings ('ferro' and 'antiferro' Mn $d e_{g}$ orderings $^{12}$ ). The preferred electronic phase appears to be strongly dependent on the $\mathrm{A}^{2+}$ and $\mathrm{R}^{3+}$ cation sizes and whether they appear disordered (such as with $\mathrm{Ca}$ and $\mathrm{La} / \mathrm{Pr}$ ) or layered (such as for $\mathrm{Ba}$ and $\mathrm{La} / \mathrm{Tb} / \mathrm{Y}^{5,10,12}$ ) in the crystal.

In this regard, it is interesting to compare the physics of the half-doped manganites, with that of the half-doped titanates. At the bulk level, the $\mathrm{A}^{2+}$ and $\mathrm{R}^{3+}$ cations are found to naturally disorder ${ }^{13,14}$ in the titanates, and typically no charge and orbitalordered Mott insulating phase is observed at half-doping ${ }^{15}$. An exception has been recently discovered for the case of very small $\mathrm{A}^{2+}$-cations, such as $\mathrm{Ca}_{0.5} \mathrm{Lu}_{0.5} \mathrm{TiO}_{3}$, where a rocksalt charge-ordered and $d_{x y} t_{2 g}$ orbital-ordered Mott insulating phase was recently proposed ${ }^{16}$. On the other hand, in layered superlattices consisting of a repeating unit of $k$ layers of $\mathrm{A}^{2+} \mathrm{TiO}_{3}$ with $l$ layers of $\mathrm{R}^{3+} \mathrm{TiO}_{3}$, exotic behaviour such as an interface two-dimensional (2D) electron gas ${ }^{17}$, which can be $\mathrm{FM}^{18}$ and superconducting ${ }^{19}$ has been reported.

Here we consider half-doped titanates in short-period [001] superlattice form $(k=l=1)$ (see Fig. 1$)$, which can in principle be artificially grown using modern layer-by-layer growth techniques (see for instance refs. 20,21 and references within). This case resembles a bulk-like double perovskite, where every $\mathrm{Ti}$ ion shares the same mixed environment at odds with thicker superlattices. A careful first principles investigation (see Methods) including all possible degrees of freedom reveals an unexpected FM and ferroelectric insulating ground state. The electronic structure exhibits an intricate orbital and charge ordering, which is argued to be at the heart of the observed ferromagnetism. A symmetry lowering structural distortion enabling this particular orbital ordering is also found to drive the ferroelectricity. The results appear rather general across the whole $\mathrm{A}^{2+} \mathrm{TiO}_{3}-\mathrm{R}^{3+} \mathrm{TiO}_{3}$ $(k=l=1)$ series, being shared by a wide variety of combination of cations with a large variation of cationic sizes. This has allowed for universal physical principles to be rationalized and new multiferroic design guidelines to be proposed. The subtle interplay between electronic and structural degrees of freedom are compared with the manganites and novel features are highlighted.

\section{Results}

Ferroelectricity. To unravel the unexpected ferroelectric and FM behaviour, we begin by focussing on the atomic structure of the $\mathrm{A}^{2+} \mathrm{TiO}_{3}-\mathrm{R}^{3}+\mathrm{TiO}_{3}$ superlattice (see Fig. 1). Unless stated otherwise the results presented throughout, although qualitatively similar across the whole series (see Supplementary Tables 3 and 4), are presented for the case of $\mathrm{SmTiO}_{3}-\mathrm{SrTiO}_{3}$. In all cases, we

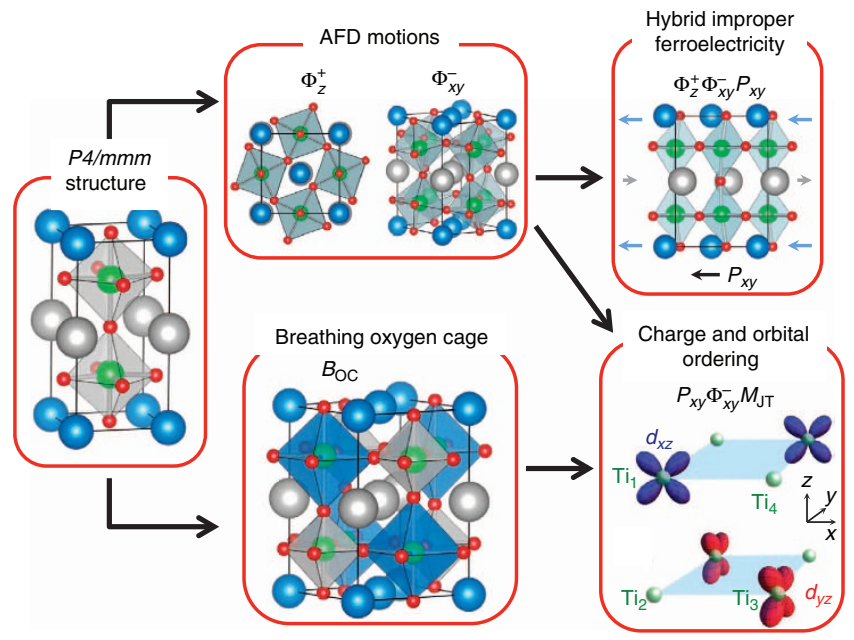

Figure 1 | Superlattice geometry, major structural distortions, and resulting ferroelectric, charge and orbital ordering. The $\mathrm{A}^{2+} \mathrm{TiO}_{3}-$ $\mathrm{R}^{3+} \mathrm{TiO}_{3}$ digital superlattice 10 -atom high-symmetry tetragonal $\mathrm{P} 4 / \mathrm{mmm}$ reference structure undergoes two major structural distortions: AFD motions and a breathing oxygen cage distortion. The rocksalt arrangement of large (blue) and small (grey) octahedral cages of the breathing distortion are shown in the 20-atom cell. The AFD motions induce ferroelectricity through a unique anharmonic coupling to an in-plane polar mode. The combination of the AFD motions and breathing oxygen cage allows for an unusual charge and orbital ordering. Blue, grey, red and green spheres represent $\mathrm{R}^{3+}, \mathrm{A}^{2+}, \mathrm{O}$ and $\mathrm{Ti}$, respectively. Distortions are exaggerated for illustrative purposes.

find a $P 2_{1}$ symmetry ground state that consists of a complex combination of several lattice distortions (see Table 1) of the high-symmetry $(P 4 / \mathrm{mmm})$ cube-on-cube double perovskite. Out of all the distortions, the largest in amplitude are oxygen octahedral rotations, both in-phase around the out-of-plane $(z)$ axis, $\Phi_{z}^{+}$and anti-phase around the in-plane ( $x$ and $y$ ) axes, $\Phi_{x y}^{z}\left(\Phi_{x}^{-}+\Phi_{y}^{-}\right)$. These so-called antiferrodistortive (AFD) motions are ubiquitous in perovskites, appearing through steric effects described by the Goldschmidt tolerance factor ${ }^{22}$. The particular AFD pattern found here, $\mathrm{a}^{-} \mathrm{a}^{-} \mathrm{c}^{+}$in Glazer's notations $^{23}$, is the most common pattern shown by perovskites $^{24}$, certainly with tolerance factors between $0.8-1.0$, the case studied here. This particular AFD pattern is stabilized over others in simple bulk perovskites through unique anharmonic couplings allowing the subsequent appearance of anti-polar A-cation motions ${ }^{25-27}$, located at the zone-boundary of the cubic $\mathrm{ABO}_{3}$ Brillouin zone. In the $\mathrm{A}^{2+} \mathrm{TiO}_{3}-\mathrm{R}^{3+} \mathrm{TiO}_{3}$ digital superlattices, this $\mathrm{A}$ - and $\mathrm{R}$-cation motion transforms to the zone-centre, becoming polar in nature. The precise form of the anharmonic coupling is trilinear, $\Phi_{z}^{+} \Phi_{x y}^{-} P_{x y}$, which is the signature of the so-called rotationally driven hybrid improper ferroelectricity ${ }^{28-32}$. Indeed, we calculate all superlattices to exhibit spontaneous polarizations in the range of 7-23 $\mu \mathrm{C} \mathrm{cm}^{-2}$ (see for example Table 1), approaching that of $\mathrm{BaTiO}_{3}$, which is among the highest reported in hybrid improper ferroelectrics. Since the $P_{x y}$ mode has a 'ferri'-like character of the A-and R-cation motion (see Fig. 1), the large polarization is achieved by maximizing the mode polarity through choosing A- and R-cations not only with asymmetric cation sizes ${ }^{33}$ but also here thanks to significantly distinct Born effective charges (or valences in the simplest picture).

Charge ordering. A necessary requirement of ferroelectricity is to be electronically insulating, which is not a priori obvious in these 
Table 1 | Key quantities for a selection of $\mathrm{A}^{2}+\mathrm{TiO}_{3}-\mathrm{R}^{2}+\mathrm{TiO}_{3}$ superlattices.

\begin{tabular}{|c|c|c|c|c|c|c|c|c|}
\hline \multirow[t]{2}{*}{$\mathbf{R}^{\mathbf{3}+}, \mathbf{A}^{\mathbf{2}+}$} & \multicolumn{5}{|c|}{$\mathbf{Q}$} & \multirow[t]{2}{*}{$P$} & \multirow[t]{2}{*}{$\Delta$} & \multirow[t]{2}{*}{$\Delta E$} \\
\hline & $\boldsymbol{\Phi}_{\mathbf{z}}^{+}$ & $\Phi_{x y}^{-}$ & $P_{x y}$ & $B_{\text {OC }}$ & $\boldsymbol{M}_{\mathrm{JT}}$ & & & \\
\hline $\mathrm{Sm}, \mathrm{Sr}$ & 0.96 & 1.19 & 0.56 & 0.10 & 0.04 & 14.9 & 0.46 & 20.1 \\
\hline $\mathrm{Y}, \mathrm{Sr}$ & 1.10 & 1.30 & 0.66 & 0.11 & 0.04 & 16.7 & 0.57 & 18.0 \\
\hline $\mathrm{Sm}, \mathrm{Ba}$ & 0.75 & 0.96 & 0.48 & 0.13 & 0.07 & 18.6 & 0.50 & 18.5 \\
\hline $\mathrm{Y}, \mathrm{Ba}$ & 0.95 & 1.08 & 0.59 & 0.14 & 0.07 & 21.2 & 0.60 & 13.9 \\
\hline $\mathrm{Tm}, \mathrm{Ba}$ & 1.05 & 1.16 & 0.65 & 0.16 & 0.07 & 23.4 & 0.66 & 10.5 \\
\hline
\end{tabular}

$A F D$, antiferrodistortive; AFM, antiferromagnetism.

Amplitude $Q(\AA)$ of lattice distortions (in-phase $\Phi_{z}^{+}$and anti-phase $\Phi_{x y}^{-}$AFD motions, polar mode $P_{x y}$ Jahn-Teller mode appearing at the $M$-point of the cubic Brillouin zone $M$ JT and breathing oxygen cage $B_{O C}$ ), polarization $P\left(\mu C \mathrm{~cm}^{-2}\right.$ ), band gap $\Delta(\mathrm{eV})$ and gain of energy for FM versus AFM solution (see Methods) per 20-atom formula unit $\Delta E$ (meV).

a

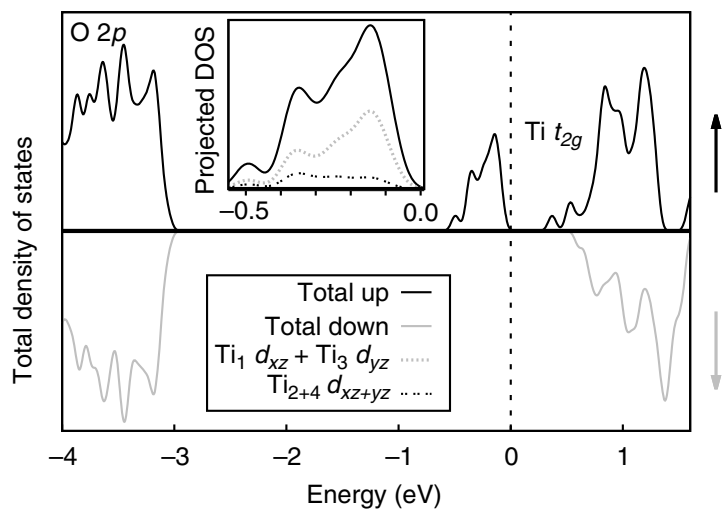

b
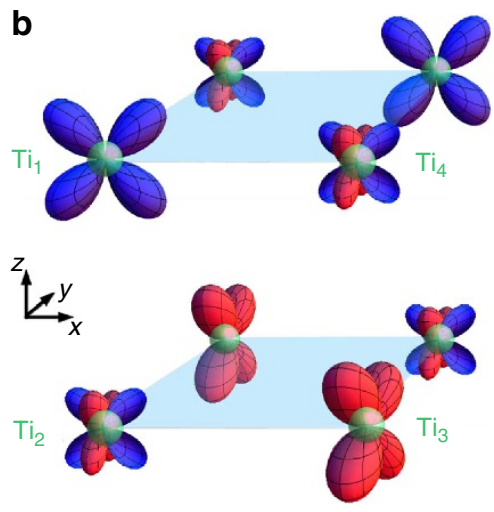

Figure 2 | Electronic structure-charge and orbital ordering. (a) Total and projected spin-polarized DOS. The upper panel (solid black), and lower panel (solid grey) correspond to up and down spins, respectively. The vertical dashed line represents the Fermi level. The Projected DOS in the inset compares the $\mathrm{Ti}_{1} d_{x z}+\mathrm{Ti}_{3} d_{y z}$ (grey fine dashed lines) with the $\mathrm{Ti}_{2+4} d_{x z+y z}$ (black dashed lines) DOS of the spin-split-off state. (b) Sketch of the resulting orbital ordering. Green spheres represent Ti sites, and red and blue lobes represent $d_{y z}$ and $d_{x z}$ orbitals, respectively.

half-doped titanates. Allowing only AFD motions yields a metastable system with the usual $P m c 2_{1}$ symmetry (equivalent to Pnma but for the superlattice). Within this symmetry, the system is metallic and all $\mathrm{Ti}$ ions share the same magnetic moment by symmetry (roughly $0.45 \mu_{\mathrm{B}}$ per $\mathrm{Ti}$ site). However, we observe an electronic instability leading to an incipient charge ordering between the four $\mathrm{Ti}$ sites. Indeed, releasing the symmetry constraint on the electronic wavefunction while keeping the atoms fixed to a $P m c 2_{1}$ structure produces an energy gain due to an incipient $d^{1}-d^{0}$ charge ordering. Relaxing the geometry produces the $P 2_{1}$ ground state with the appearance of a related breathing $B_{\mathrm{OC}}$ motion, which expands or contracts the oxygen octahedra in nearest neighbour unit cells (see Fig. 1 and Table 1). This breathing distortion does not lift the degeneracy of the $t_{2 g}$ levels of the $\mathrm{Ti}$ atoms at the centre of each octahedra, but does lift the degeneracy between Ti sites, amplifying the charge ordering, and helping render the superlattices insulating. The charge ordering mimics the rocksalt pattern, and hence appears at the $R$-point ( $M$-point) of the cubic (tetragonal) Brillouin zone, maximizing the distance between the more highly charged $d^{0} \mathrm{Ti}^{4+}$ ions. Please see Supplementary Note 1, and associated Supplementary Tables 6 and 8, for further discussion on the breathing mode and charge ordering.

Orbital ordering. This charge-ordered insulating state is indeed found, as indicated through the spin-resolved density of states (DOS), as presented for the FM solution in Fig. 2 (for the full projected DOS see Supplementary Fig. 1). The states near the Fermi level exhibit Ti $d$ character, while the O $2 p$ states appear deeper into the valence. A band gap separates an occupied spinpolarized 'split-off band from the remaining unoccupied Ti $d$ conduction band. This 'split-off valence is found to consist of 2 bands with the majority of weight located at 2 different Ti sites, out of the 4 possible Ti sites in total in the 20 -atom unit cell (see Fig. 1). These two sites are surrounded by the expanded oxygen octahedra, which we label the $\mathrm{Ti} d^{1}$ sites, as opposed to the two other Ti $d^{0}$ sites surrounded by a contracted oxygen octahedra. Interestingly the orbital occupation of the two $d^{\mathrm{P}}$ sites are different with one showing $d_{x z}$ and the other $d_{y z}$ character. The resulting orbital ordering corresponds to the same pattern, albeit with half the sites empty, as that achieved through a Jahn-Teller distortion appearing at the $M$-point of the cubic Brillouin zone $\left(M_{\mathrm{JT}}\right)$. This lattice distortion is indeed observed in the ground state (see Table 1). In fact it is found that the AFD motion themselves can achieve this orbital ordering, in absence of the $M_{\mathrm{JT}}$, by lowering the symmetry of the bulk (superlattice) cubic (tetragonal) reference phase to orthorhombic (monoclinic). Indeed, similarly to the improper appearance of the polar A-cation motions, the AFD motions also drive the appearance of the $M_{\mathrm{JT}}$ distortion through another trilinear coupling, $P_{x y} \Phi_{x y}^{-} M_{\mathrm{JT}}^{34}$. The fact that the AFD motions produce the same orbital ordering as the $M_{\mathrm{JT}}$ distortion is simply a consequence of this symmetry relation.

Interestingly, we find that the charge ordering is not exactly the ideal case of $d^{1}-d^{0}$. Defining $m$ as the magnitude of the local magnetic moment, we find a $d^{(1-m)}-d^{m}$ ordering, with a significant weight on the nominally $d^{0}$ sites $(0.11<m<0.18$ depending on the A- and R-species), displaying a mixed $d_{x z}-d_{y z}$ 


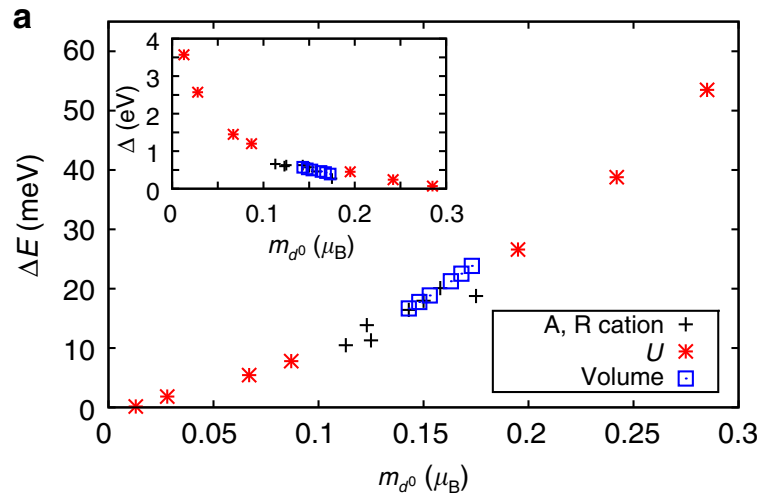

b

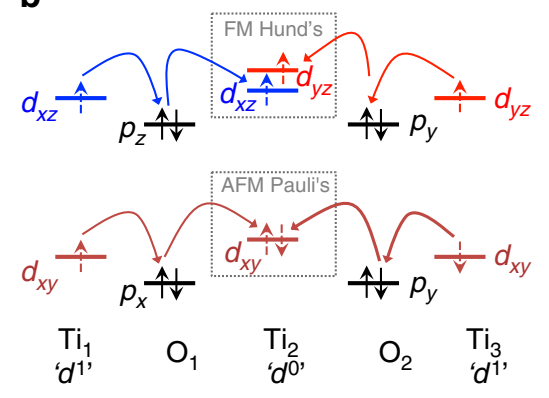

Figure 3 I Insights into magnetic interactions. (a) Energy gain of FM compared with AFM state (see Methods) per 20 -atom formula unit, $\Delta E$, as a function of the moment on the $d^{0}$ sites, $m_{d^{0}}$. Inset: band gap $\Delta$ versus $m_{d^{0}}$. The different points correspond to various perturbations of the superlattice, including varying the $A$ - and R-cation species, the on-site Coulomb repulsion $U$ and the volume of the cell. (b) Simplified spin exchange diagram between nearest neighbour $d^{1}$ sites $\left(\mathrm{Ti}_{1}\right.$ and $\mathrm{Ti}_{3}$ ) via a $d^{0}$-site $\left(\mathrm{Ti}_{2}\right)$. The curved arrows represent inter-site spin hopping through oxygen ions, and the dashed box represents intra-site spin-exchange via Hund's rules, in the case of $d_{x z}-d_{y z}$ orbital ordering due to AFD motions, or Pauli's exclusion principle, in the case of $d_{x y}$ orbital ordering in the absence of AFD motions.

character. The system remains insulating despite this delocalized state, through a strong hybridization of the two occupied Ti $d$ bands. The resulting intricate and entangled charge and orbital ordering is sketched in Fig. 2.

Magnetic ordering. Having discussed the lattice, charge and orbital degrees of freedom, and their coupling, we move to the final degree of freedom-spin. Various magnetic orderings (see methods and Supplementary Tables 4 and 5) were studied, and in all cases and chemistries the FM state was unexpectedly found to be the lowest in energy. In an attempt to understand the origin of this rare insulating FM ground state, we observe a universal trend across $\mathrm{A}^{2+} \mathrm{TiO}_{3}-\mathrm{R}^{3+} \mathrm{TiO}_{3}$ superlattices with all A- and $\mathrm{R}$-species and various applied perturbations. This trend is shown in Fig. 3, where it is seen that the energy difference between AFM and FM states, or more simply the strength of the FM exchange, is clearly strongly dependent on the spin density of $d$ electrons on the nominally $d^{0}$ sites. Indeed, as the system tends to the ideal charge ordering $d^{1}-d^{0}$, the FM and AFM energies tend to equilibrate. Therefore, in this regime, the spins on the two $d^{1}$ sites are completely decoupled. However, as the $d^{0}$-site electrons become populated, the FM exchange strengthens. This key observation indicates that the FM exchange mechanism relies on a real intrasite spin exchange on the nominally $d^{0}$ sites, rather than a virtual direct exchange between $d^{1}$ sites. We propose the intra-site FM spin exchange as Hund's rule (see Fig. 3 ).

The explanation of the FM interaction between $d^{1}$ sites relies on two simple arguments, sketched in Fig. 3 (for simplicity in the following, we only consider the four $\mathrm{Ti}$ sites of Fig. 2, but the same arguments hold when including all neighbours in 3D). First, due to covalency effects, the electron on the $\mathrm{Ti}_{1}\left(d^{1}\right)$ site delocalizes partly on the neighbouring $\mathrm{Ti}_{2}$ and $\mathrm{Ti}_{4}\left(d^{0}\right)$ sites necessarily with the same spin (since it is the same electron) and the analogous occurs to the electron on the $\mathrm{Ti}_{3}\left(d^{1}\right)$ site. Second, due to the orbital ordering, the charges on $\mathrm{Ti}_{2}$ (and equivalently $\mathrm{Ti}_{4}$ ) that originate independently from $\mathrm{Ti}_{1}$ and $\mathrm{Ti}_{3}$ populate a different orbital ( $d_{x z}$ and $d_{y z}$ respectively). In the same spirit as Hund's rule of maximum multiplicity, which maximizes the spin of an electron configuration of an atom, a FM solution of the $d_{x z}^{0}-d_{y z}^{0}$ spins becomes the ground state. This rule minimizes the intra-site Coulombic energy. The FM solution relies on both kinetic and Coulomb contributions, to delocalize the spinpolarized $d^{1}$ electrons partially on the $d^{0}$ sites and to align the $d^{0}$ intra-site spins, respectively. In this regard, it resembles a double (kinetic + Coulomb) exchange, even if in an insulator. This FM mechanism in $d^{0}-d^{1}$ charge-ordered systems, which has been previously overlooked, extends the list of $d^{i}-d^{j}$ charge orderings considered within the Goodenough-Kanamori rules $8,35,36$.

We performed two computational experiments to further support this picture. In the first computational experiment, we took the ground state structure and artificially modified the magnitude of the $M_{\mathrm{TT}}$ distortion (leaving all other lattice modes unchanged). Eventually at a huge $M_{\mathrm{JT}}$ distortion (50 times larger than the ground state magnitude, but similar in size to the prototypical Jahn-Teller system $\mathrm{LaMnO}_{3}$ ), the $d_{x z}^{0}-d_{y z}^{0}$ splitting (see Fig. 3) is so large that only one of the orbitals becomes populated at each $d_{0}$-site, removing the Hund's interaction and producing an AFM ground state. In the second case, the AFD motions were artificially neglected (along with all other motions except $\left.\mathrm{B}_{\mathrm{OC}}\right)$. The tetragonal symmetry can still lead to an insulating charge-ordered state but now with $d_{x y}$ orbital ordering, once an unrealistically large on-site Coulomb repulsion $U$ $(>7 \mathrm{eV})$ is enforced ${ }^{37}$. We have reproduced this constraint and find that the AFM solution now becomes the ground state. This can again be understood through the intra-site spin exchange on the nominally $d^{0}$ sites (see Fig. 3 ). An anti-alignment of spins on the intra-site $d_{x y}^{0}-d_{x y}^{0}$ orbitals (and hence on the inter-site $d_{x y}^{1}-d_{x y}^{1}$ orbitals) is favoured from Pauli's exclusion principlesnow that the same orbital (spacial coordinate) is occupied, opposite electron spins are favoured. Therefore the combination of charge ordering at the $R$-point, via breathing oxygen cage motions, and orbital ordering at the $M$-point, via AFD and $M_{\mathrm{JT}}$ motions (but not too large), of the cubic Brillouin zone are the key to realize the FM ground state.

\section{Discussion}

We note the FM mechanism here is distinct from the FM behaviour found in some $\mathrm{R}^{3+} \mathrm{TiO}_{3}$ bulk compounds (for $\mathrm{R}$ from $\mathrm{Gd}$ to $\mathrm{Yb}$ ). To illustrate, for example, we note the FM coupling becomes stronger as the $\mathrm{A}$ size decreases and band gap ${ }^{38}$ increases in bulk $\mathrm{R}^{3+} \mathrm{TiO}_{3}$ compounds. This is seen through a transition from AFM to FM and then a rise in FM $T_{\mathrm{C}}$ with decreasing $\mathrm{A}$ size $\mathrm{e}^{39}$. The reverse trend is observed here, where the FM coupling strengthens with increasing A-cation size and decreasing band gap. 
It is interesting finally to compare the FM solution of the halfdoped titanates, to the (A-type or E-type) AFM solution of the half-doped manganites. The magnetic ordering is likely different due to the superexchange interaction in the manganites between the half-filled core $t_{2 g}$ electrons on every $\mathrm{Mn}$ site. This superexchange path is clearly cutoff by the rocksalt arrangement of $d^{0}$ sites in the titanates studied here. A FM insulating phase of the manganites does exist for the specific case of $\operatorname{Pr}_{1-x} \mathrm{Ca}_{x} \mathrm{MnO}_{3}$ with doping $x \approx 0.2$ (refs 40-42), where a FM exchange wins due to a particular $e_{g}$ orbital polaron ordering ${ }^{43}$, not completely dissimilar to the $t_{2 g}$ case here, using the Goodenough-Kanamori rules $^{8,35,36}$.

Having rationalized the key concepts underlying the FM and ferroelectric behaviour, we can propose some basic design rules and suggest alternative materials to achieve a similar multiferroic state. To ensure an insulating ground state, the smaller the A- and $\mathrm{R}$-cations the larger the band gap, consistent with the recent observation of insulating $\mathrm{Ca}_{0.5} \mathrm{Lu}_{0.5} \mathrm{TiO}_{3}$ (ref. 16). Maximizing the ferroelectric polarization can be achieved by choosing the greatest asymmetry in both the Born charge and size of the A- and R-cations. Regarding ferromagnetism, the exchange coupling constant $J$, and hence the FM $T_{C}$, increases the greater the delocalization of charges onto the nominally $d^{0}$ sites, which can be altered with strain and A-site chemistry as shown in Fig. 3. It could be interesting to test the same concepts on other B-cations such as the $4 d$ and $5 d$ transition metals, $d^{9}-d^{10}$ systems such as the cuprates, which can be viewed as a $d^{1}-d^{0}$ hole charge ordering, or $d^{6}-d^{7}$ systems with ordering of the single $e_{g}$ electron instead of the $t_{2 g}$ in the titanates. Here we might expect a similar type of entangled charge and orbital orderings leading to unexpected ferromagnetism. We hope that this study might encourage the search for related novel electronic phases within these systems.

\section{Methods}

First principles calculations. Here we describe a three-step first principles strategy for the titanate calculations. (i) Initially hybrid functional calculations, using the B1WC functional ${ }^{44}$ within the Crystal code ${ }^{45}$, were performed on bulk titanates $\left(\mathrm{YTiO}_{3}, \mathrm{LaTiO}_{3}, \mathrm{SrTiO}_{3}\right.$ and $\left.\mathrm{BaTiO}_{3}\right)$ and compared with experiment (see Supplementary Table 1). The details of the pseudopotentials and basis sets for $\mathrm{La}, \mathrm{Sr}, \mathrm{Ti}$ and $\mathrm{O}$ can be found in ref. 46 , while for $\mathrm{Ba}$ and $\mathrm{Y}$ details can be found in refs. 47,48 respectively. (ii) Once verified on the bulk, hybrid functional calculations were performed on several representative $\mathrm{R}^{3}+\mathrm{TiO}_{3}-\mathrm{A}^{2}+\mathrm{TiO}_{3}$ superlattices. Ground states were determined through condensing various lattice instabilities and recalculating phonon frequencies. Band gaps and FM and AFM energies were computed on the ground states. (iii) To make the calculations computationally tractable, and allow simulation of many more chemistries, $\mathrm{GGA}+\mathrm{U}$ calculations ${ }^{49}$, using the PBEsol functional ${ }^{50}$ and projector augmented wave potentials within the VASP $\operatorname{code}^{51}$, were performed on the relaxed hybrid functional superlattice ground state structures. The on-site Coulomb repulsion $U$ was fitted to simultaneously reproduce the band gap and spin flip energy of the hybrid functionals, and minimize atomic forces (see Supplementary Table 2), with a value of $U=3.0 \mathrm{eV}$ found to be appropriate. This allowed for full structural relaxation using GGA $+\mathrm{U}$ on a wide-range of $\mathrm{R}^{3+} \mathrm{TiO}_{3}-\mathrm{A}^{2+} \mathrm{TiO}_{3}$ structures $\left(\mathrm{R}^{3+}\right.$ : La, Pr, Sm, Y, Tm, Lu; $\mathrm{A}^{2+}: \mathrm{Sr}, \mathrm{Ba}, \mathrm{Ca}$ and we also include $\mathrm{Eu}^{2+}$ - see Supplementary Table 7 ). We used a $5 \times 5 \times 3$ Monkhorst-Pack k-point mesh to model the 20-atom cell and a plane wave cutoff of $500 \mathrm{eV}$. All lattice vectors were fully relaxed. Small to moderate in-plane strains resembling thin film epitaxy were not found to alter the qualitative findings presented. Structural optimizations were performed until the difference of forces were $<10^{-6} \mathrm{eV} \AA^{-1}$ and the energy difference between conjugate gradient steps became lower $<10^{-8} \mathrm{eV}$. Note: we do not consider $\mathrm{R} f$-electrons explicitly to simplify calculations, since in practice the 4 -electrons order at much lower temperatures than the $3 d$-electrons. We have tested explicitly including $f$-electrons and find that the results are not affected. Unless stated otherwise the AFM ordering presented is the simplest AFM configuration between first-neighbour $d^{1}$ sites allowed within the $\sqrt{2} \times \sqrt{2} \times 1$ 20 -atom cell. For longer range AFM orderings $2 \times 2 \times 1$ and $2 \sqrt{2} \times \sqrt{2} \times 1,40$-atom supercells were relaxed with all possible spin orderings considered, including the CE-type. AFM orderings requiring cell doubling out-of-plane ( 80 atoms) were additionally checked (see Supplementary Table 5). Atomic positions were relaxed for all AFM orderings. The polarization was computed using the Berry phase approach as implemented in VASP. The amplitude $Q(\AA)$ of lattice distortions, was determined through the symmetry-mode analysis using the AMPLIMODES software ${ }^{52}$.

\section{References}

1. Zubko, P., Gariglio, S., Gabay, M., Ghosez, P. \& Triscone, J.-M. Interface physics in complex oxide heterostructures. Annu. Rev. Condens. Matter Phys. 2, 141-165 (2011).

2. Hwang, H. et al. Emergent phenomena at oxide interfaces. Nat. Mater. 11, 103-113 (2012).

3. Tokura, Y. \& Nagaosa, N. Orbital physics in transition-metal oxides. Science 288, 462-468 (2000).

4. Dagotto, E. Complexity in strongly correlated electronic systems. Science 309, 257-262 (2005).

5. Millange, F., Caignaert, V., Domenges, B., Raveau, B. \& Suard, E. Orderdisorder phenomena in new $\mathrm{LaBaMn}_{2} \mathrm{O}_{6-x} \mathrm{CMR}$ perovskites. Crystal and magnetic structure. Chem. Mater. 10, 1974-1983 (1998).

6. Millange, F., Suard, E., Caignaert, V. \& Raveau, B. $\mathrm{YBaMn}_{2} \mathrm{O}_{5}$ : crystal and magnetic structure reinvestigation. Mater. Res. Bull. 34, 1-9 (1999).

7. Nakajima, T., Kageyama, H. \& Ueda, Y. Successive phase transitions in a metal-ordered manganite perovskite $\mathrm{YBaMn}_{2} \mathrm{O}_{6}$. J. Phys. Chem. Solids 63, 913-916 (2002).

8. Goodenough, J. B. Theory of the role of covalence in the perovskite-type manganites [La,M(II)] $\mathrm{MnO}_{3}$. Phys. Rev 100, 564 (1955).

9. Wollan, E. \& Koehler, W. Neutron diffraction study of the magnetic properties of the series of perovskite-type compounds $\left[\mathrm{La}_{1-x} \mathrm{Ca}_{x}\right] \mathrm{MnO}_{3}$. Phys. Rev. 100, 545 (1955).

10. Williams, A. J. \& Attfield, J. P. Alternative charge-order polymorph for half-doped manganites in $\mathrm{TbBaMn}_{2} \mathrm{O}_{6}$. Phys. Rev. B 66, 220405 (2002).

11. Radaelli, P., Cox, D., Marezio, M. \& Cheong, S. Charge, orbital, and magnetic ordering in $\mathrm{La}_{0.5} \mathrm{Ca}_{0.5} \mathrm{MnO}_{3}$. Phys. Rev. B 55, 3015 (1997).

12. Williams, A. J. \& Attfield, J. P. Ferro-orbital order in the charge-and cationordered manganite $\mathrm{YBaMn}_{2} \mathrm{O}_{6}$. Phys. Rev. B 72, 024436 (2005).

13. Ju, H., Eylem, C., Peng, J., Eichhorn, B. \& Greene, R. Metal-insulator transitions and weak ferromagnetism in the electron-correlated system $\mathrm{Nd}_{1-x} \mathrm{Ca}_{x} \mathrm{TiO}_{3}$. Phys. Rev. B 49, 13335 (1994).

14. Eylem, C. et al. Structural, magnetic, and transport properties of $\mathrm{R}_{x} \mathrm{Ba}_{1-x} \mathrm{TiO}_{3-\delta}$ solid solutions, where $\mathrm{R}=\mathrm{La}, \mathrm{Nd}, \mathrm{Gd}$, Er, and Y: rare-earthdependent metal-to-semiconductor transitions. Chem. Mater. 4, 1038-1046 (1992).

15. Tokura, Y. et al. Filling dependence of electronic properties on the verge of metal-Mott-insulator transition in $\mathrm{Sr}_{1-x} \mathrm{La}_{x} \mathrm{TiO}_{3}$. Phys. Rev. Lett. 70, 2126 (1993).

16. Komarek, A. et al.Evidence for charge and orbital order in the doped titanates $\mathrm{RE}_{1-x} \mathrm{Ca}_{x} \mathrm{TiO}_{3}(\mathrm{RE}=\mathrm{Y}, \mathrm{Er}, \mathrm{Lu})$ arXiv preprint arXiv:1109.0234 (2011).

17. Ohtomo, A., Muller, D., Grazul, J. \& Hwang, H. Y. Artificial chargemodulationin atomic-scale perovskite titanate superlattices. Nature 419, 378-380 (2002).

18. Okamoto, S. \& Millis, A. J. Electronic reconstruction at an interface between a mott insulator and a band insulator. Nature 428, 630-633 (2004).

19. Biscaras, J. et al. Two-dimensional superconductivity at a Mott insulator/band insulator interface $\mathrm{LaTiO}_{3} / \mathrm{SrTiO}_{3}$. Nat. Commun. 1, 89 (2010).

20. Martin, L., Chu, Y.-H. \& Ramesh, R. Advances in the growth and characterization of magnetic, ferroelectric, and multiferroic oxide thin films. Mat. Sci. Eng. R-Rep. 68, 89-133 (2010).

21. Martin, L. W. \& Schlom, D. G. Advanced synthesis techniques and routes to new single-phase multiferroics. Curr. Opin. Solid State Mater. Sci. 16, 199-215 (2012).

22. Goldschmidt, V. M. Die gesetze der krystallochemie. Naturwissenschaften 14, 477-485 (1926).

23. Glazer, A. The classification of tilted octahedra in perovskites. Acta Crystallogr. B 28, 3384-3392 (1972).

24. Lufaso, M. W. \& Woodward, P. M. Prediction of the crystal structures of perovskites using the software program spuds. Acta Crystallogr. B 57, 725-738 (2001).

25. Miao, N., Bristowe, N. C., Xu, B., Verstraete, M. J. \& Ghosez, P. First-principles study of the lattice dynamical properties of strontium ruthenate. J. Phys.: Condens. Matter 26, 035401 (2014).

26. Thomas, N. W. The compositional dependence of octahedral tilting in orthorhombic and tetragonal perovskites. Acta Crystallogr. B 52, 16-31 (1996).

27. Woodward, P. M. Octahedral tilting in perovskites. ii. structure stabilizing forces. Acta Crystallogr. B 53, 44-66 (1997).

28. Bousquet, E. et al. Improper ferroelectricity in perovskite oxide artificial superlattices. Nature 452, 732-736 (2008).

29. Benedek, N. A. \& Fennie, C. J. Hybrid improper ferroelectricity: a mechanism for controllable polarization-magnetization coupling. Phys. Rev. Lett. 106, 107204 (2011).

30. Fukushima, T., Stroppa, A., Picozzi, S. \& Perez-Mato, J. M. Large ferroelectric polarization in the new double perovskite $\mathrm{NaLaMnWO}_{6}$ induced by non-polar instabilities. Phys. Chem. Chem. Phys. 13, 12186-12190 (2011). 
31. Benedek, N. A., Mulder, A. T. \& Fennie, C. J. Polar octahedral rotations a path to new multifunctional materials. J. Solid State Chem. 195, 11-20 (2012).

32. Rondinelli, J. M. \& Fennie, C. J. Octahedral rotation-induced ferroelectricity in cation ordered perovskites. Adv. Mater. 24, 1961-1968 (2012).

33. Mulder, A. T., Benedek, N. A., Rondinelli, J. M. \& Fennie, C. J. Turning $\mathrm{ABO}_{3}$ antiferroelectrics into ferroelectrics: design rules for practical rotation-driven ferroelectricity in double perovskites and $\mathrm{A}_{3} \mathrm{~B}_{2} \mathrm{O}_{7}$ ruddlesden-popper compounds. Adv. Funct. Mater. 23, 4810-4820 (2013).

34. Varignon, J., Bristowe, N. C., Bousquet, E. \& Ghosez, P. Coupling and electrical control of structural, orbital and magnetic orders in perovskites. arXiv preprint arXiv:1409.8422 (2014).

35. Goodenough, J. B. An interpretation of the magnetic properties of the perovskite-type mixed crystals $\mathrm{La}_{1-} \mathrm{Sr}_{x} \mathrm{CoO}_{3}-\lambda$. J. Phys. Chem. Solids 6, 287-297 (1958).

36. Kanamori, J. Superexchange interaction and symmetry properties of electron orbitals. J. Phys. Chem. Solids 10, 87-98 (1959).

37. Pentcheva, R. \& Pickett, W. E. Correlation-driven charge order at the interface between a mott and a band insulator. Phys. Rev. Lett. 99, 016802 (2007).

38. Okimoto, Y., Katsufuji, T., Okada, Y., Arima, T. \& Tokura, Y. Optical spectra in $(\mathrm{La}, \mathrm{Y}) \mathrm{TiO}_{3}$ : variation of Mott-Hubbard gap features with change of electron correlation and band filling. Phys. Rev. B 51, 9581 (1995).

39. Komarek, A. et al. Magnetoelastic coupling in $\mathrm{RTiO}_{3}(\mathrm{R}=\mathrm{La}, \mathrm{Nd}, \mathrm{Sm}, \mathrm{Gd}, \mathrm{Y})$ investigated with diffraction techniques and thermal expansion measurements. Phys. Rev. B 75, 224402 (2007).

40. Jirak, Z., Krupička, S., Šimša, Z., Dlouha, M. \& Vratislav, S. Neutron diffraction study of $\mathrm{Pr}_{1-x} \mathrm{Ca}_{x} \mathrm{MnO}_{3}$ perovskites. J. Magn. Magn. Mater. 53, 153-166 (1985).

41. Tomioka, Y., Asamitsu, A., Moritomo, Y., Kuwahara, H. \& Tokura, Y. Collapse of a charge-ordered state under a magnetic field in $\mathrm{Pr}_{1 / 2} \mathrm{Sr}_{1 / 2} \mathrm{MnO}_{3}$. Phys. Rev. Lett. 74, 5108 (1995).

42. Yoshizawa, H., Kawano, H., Tomioka, Y. \& Tokura, Y. Neutron-diffraction study of the magnetic-field-induced metal-insulator transition in $\mathrm{Pr}_{0.7} \mathrm{Ca}_{0.3} \mathrm{MnO}_{3}$. Phys. Rev. B 52, R13145 (1995).

43. Mizokawa, T., Khomskii, D. \& Sawatzky, G. Orbital polarons and ferromagnetic insulators in manganites. Phys. Rev. B 63, 024403 (2000).

44. Bilc, D. et al. Hybrid exchange-correlation functional for accurate prediction of the electronic and structural properties of ferroelectric oxides. Phys. Rev. B 77, 165107 (2008).

45. Dovesi, R. et al. Crystal: a computational tool for the ab initio study of the electronic properties of crystals. Z. Kristallogr. 220, 571-573 (2005).

46. Delugas, P. et al. Spontaneous 2-dimensional carrier confinement at the n-type $\mathrm{SrTiO}_{3} / \mathrm{LaAlO}_{3}$ interface. Phys. Rev. Lett. 106, 166807 (2011).

47. Piskunov, S., Heifets, E., Eglitis, R. \& Borstel, G. Bulk properties and electronic structure of $\mathrm{SrTiO}_{3}, \mathrm{BaTiO}_{3}, \mathrm{PbTiO}_{3}$ perovskites: an ab initio HF/DFT study. Comp. Mat. Sci. 29, 165-178 (2004).
48. Varignon, J., Petit, S., Gellé, A. \& Lepetit, M. B. An ab initio study of magnetoelectric coupling of $\mathrm{YMnO}_{3}$. J. Phys.: Condens. Matter 25, 496004 (2013).

49. Liechtenstein, A., Anisimov, V. \& Zaanen, J. Density-functional theory and strong interactions: orbital ordering in Mott-Hubbard insulators. Phys. Rev. B 52, 5467 (1995).

50. Perdew, J. P. et al. Restoring the density-gradient expansion for exchange in solids and surfaces. Phys. Rev. Lett. 100, 136406 (2008).

51. Kresse, G. \& Furthmüller, J. Efficient iterative schemes for ab initio total-energy calculations using a plane-wave basis set. Phys. Rev. B 54, 11169 (1996).

52. Orobengoa, D., Capillas, C., Aroyo, M. I. \& Perez-Mato, J. M. Amplimodes: symmetry-mode analysis on the bilbao crystallographic server. J. Appl. Crystallogr. 42, 820-833 (2009).

\section{Acknowledgements}

We acknowledge stimulating discussions with N. Spaldin and C. Ederer and support from the ARC project TheMoTherm and FNRS project HiT4FiT. Ph.G. acknowledges Research Professorship from the Francqui foundation and N.C.B. a Research Fellowship from the Royal Commission for the Exhibition of 1851. Calculations have been performed within the PRACE projects TheoMoMuLaM and TheDeNoMo. They also took advantage of the Céci-HPC facilities funded by F.R.S.-FNRS (Grant No 2.5020.1) and the Tier-1 supercomputer of the Fédération Wallonie-Bruxelles funded by the Walloon Region (grant no 1117545).

\section{Author contributions}

All authors contributed extensively to the work presented in this paper.

\section{Additional information}

Supplementary Information accompanies this paper at http://www.nature.com/ naturecommunications

Competing financial interests: The authors declare no competing financial interest.

Reprints and permission information is available online at http://npg.nature.com/ reprintsandpermissions/

How to cite this article: Bristowe, N. C. et al. Ferromagnetism induced by entangled charge and orbital orderings in ferroelectric titanate perovskites. Nat. Commun. 6:6677 doi: $10.1038 /$ ncomms7677 (2015)

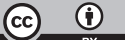

This work is licensed under a Creative Commons Attribution 4.0 International License. The images or other third party material in this article are included in the article's Creative Commons license, unless indicated otherwise in the credit line; if the material is not included under the Creative Commons license, users will need to obtain permission from the license holder to reproduce the material. To view a copy of this license, visit http://creativecommons.org/licenses/by/4.0/ 